\title{
Dapagliflozin and Empagliflozin Ameliorate Hepatic Dysfunction Among Chinese Subjects with Diabetes in Part Through Glycemic Improvement: A Single- Center, Retrospective, Observational Study
}

\author{
Paul Chi Ho Lee · Yunjuan Gu • Man Yi Yeung • Carol Ho Yi Fong • \\ Yu Cho Woo · Wing Sun Chow · Kathryn Tan · Karen Siu Ling Lam
}

Received: November 1, 2017 / Published online: January 10, 2018

(C) The Author(s) 2018. This article is an open access publication

\section{ABSTRACT}

Introduction: Sodium-glucose co-transporter 2 inhibitors (SGLT2i) improve hepatic dysfunction, although studies focusing on their underlying mechanisms are lacking, especially ones

Paul Chi Ho Lee and Yunjuan Gu are co-first authors of this article.

Enhanced content To view enhanced content for this article go to http://www.medengine.com/Redeem/ 6FFCF0604C029857.

Electronic supplementary material The online version of this article (https://doi.org/10.1007/s13300017-0355-3) contains supplementary material, which is available to authorized users.

P. C. H. Lee - M. Y. Yeung - C. H. Y. Fong ·

Y. C. Woo - W. S. Chow · K. Tan · K. S. L. Lam (ه) Department of Medicine, University of Hong Kong, Hong Kong, SAR, China

e-mail: ksllam@hku.hk

Y. Gu

Department of Endocrinology and Metabolism, Affiliated Hospital of Nantong University, Nantong, China

P. C. H. Lee · K. Tan · K. S. L. Lam

Research Centre of Heart, Brain, Hormone and Healthy Aging, University of Hong Kong, Hong Kong, SAR, China

K. S. L. Lam

State Key Laboratory of Pharmaceutical Biotechnology, University of Hong Kong, Hong Kong, SAR, China on dapagliflozin and empagliflozin. Here, we investigated the relationship between amelioration of hepatic dysfunction and improvement in various metabolic parameters among Chinese subjects with type 2 diabetes (T2DM).

Methods: This was a single-center, retrospective, observational study that involved 115 Chinese participants with T2DM treated with either dapagliflozin or empagliflozin for at least 6 months between July 2016 and February 2017. Results: Of the 115 participants included in this study, 69 received dapagliflozin and 46 received empagliflozin. After 6 months of treatment, all patients showed significant improvements in body weight (BW), systolic blood pressure (SBP) and fasting glucose (FG) and glycated hemoglobin (HbA1c) levels. All participants also showed a significant reduction in serum alanine aminotransferase (ALT) levels, from $40.3 \pm 28.0$ to $29.0 \pm 14.1 \mathrm{U} / \mathrm{L}(p<0.001)$. Pearson's correlation analysis revealed a positive correlation between the reduction in ALT levels after treatment with the respective SGLT2i and changes in FG $(p=0.014)$ and HbA1c $(p=0.043)$ levels over 6 months, but not with changes in BW and SBP. Multiple linear regression analysis revealed that the reduction in serum ALT levels was independently associated with changes in both HbA1c and FG but not with the changes in the other clinical variables, including BW.

Conclusions: Dapagliflozin and empagliflozin improved both metabolic and hepatic dysfunction as a class effect. The amelioration of 
hepatic dysfunction was mediated partly through an alleviation of hyperglycemia and possibly through an improvement in insulin resistance, independent of BW.

Keywords: Fatty liver; SGLT2 inhibitors; Type 2 diabetes mellitus

\section{INTRODUCTION}

Both non-alcoholic fatty liver disease (NAFLD) and type 2 diabetes mellitus (T2DM) are common health problems worldwide, including China [1, 2]. Strikingly, a recent study found that almost half of Chinese adults suffered from T2DM or prediabetes [2]. In another study, the prevalence of NAFLD was reported to be as high as 70\% among Chinese subjects with T2DM [3]. Notably, the relationship between T2DM and NAFLD is bi-directional: the presence of NAFLD has been related to increased risk of all-cause mortality among subjects with T2DM [4], while T2DM is also a predictor for the development, progression and complications of NAFLD. T2DM has been found to increase the risk of cirrhosis [5], double the risk of hepatocellular carcinoma [6] and to be an independent predictor of overall and liver mortality in patients with NAFLD $[4,7]$. Collectively, NAFLD has gradually emerged as an important diabetic complication.

The introduction of sodium-glucose cotransporter 2 inhibitors (SGLT2i) into clinical practice represents a novel approach to glycemic control in T2DM [8]. The benefits of this class of prescription medicines on cardiovascular and renal outcomes in patients with T2DM have been well demonstrated in large long-term randomized controlled trials [1,9-11], as well as in a large-scale real world observational study [12]. A recent review also suggested that SGLT2i are anti-diabetic agents that should be considered in the treatment of subjects comorbid with T2DM and NAFLD [13]. Although a meta-analysis of 43 randomized controlled trials showed that SGLT2i decreases serum alanine aminotransferase (ALT) levels [14], the relationship between such ALT reductions and the improvement in metabolic parameters after the use of SGLT2i remains controversial. For example, a recent pooled analysis of data from six controlled studies on canagliflozin attributed the significant improvement in serum ALT levels to a combined reduction in glycated hemoglobin (HbA1c) levels and body weight (BW) reduction [15], while the authors of some animal and human studies of other SGLT2i attributed the improvement to mechanisms independent of BW reduction $[16,17]$. Therefore, in order to examine whether SGLT2i ameliorate hepatic dysfunction through glycemic improvement and BW reduction as a class effect, we performed this retrospective observational study to investigate the relationship between improvement in hepatic dysfunction and metabolic parameters, in Chinese subjects with T2DM who were treated with either the SGLT2i dapagliflozin or empagliflozin.

\section{METHODS}

\section{Subjects}

The study included subjects with T2DM who were followed up at the Diabetes Clinic of Queen Mary Hospital, Hong Kong. All subjects were on SGLT2i, either dapagliflozin or empagliflozin, between 1 July 2016 and 28 February 2017 and were identified through the computer-based clinical management system. Only those who had been prescribed SGLT2i (dapagliflozin or empagliflozin) for at least 6 months were included in this study. Participants who were known hepatitis B carriers were excluded from this study. This study is a clinical audit and does not involve any new studies of human subjects performed by any of the authors.

\section{Design}

This was a single-center, retrospective, observational study conducted at a university hospital in Hong Kong. This study was a clinical audit consequently does not involve any new studies of human subjects performed by any of the authors. Moreover, given the clinical audit nature of the study, informed consent was not obtained from the included participants; nonetheless all 
patient records and information were rendered anonymous prior to analysis. Clinical data, including demographics (age, sex and smoking habit), anthropometric parameters [BW, height, body mass index (BMI), systolic and diastolic blood pressure (SBP and DBP) measurements], comorbidities, metabolic parameters, including fasting glucose, lipid profile, $\mathrm{HbA1c}$, and biochemical parameters, including serum creatinine (SCr), ALT, aspartate aminotransferase (AST) and albumin levels, were measured at baseline and after 26 weeks of SGLT2i use.

In this study, the estimated glomerular filtration rate (eGFR) was calculated using the Chronic Kidney Disease Epidemiology Collaboration (CKD-EPI) equation and expressed as $\mathrm{eGFR}=141 \times \min \quad(\mathrm{SCr} / \kappa, 1)^{\alpha} \times \max \quad(\mathrm{SCr} /$ $\kappa, 1)^{-1.209} \times 0.993^{\text {age }}(\times 1.018$ if female $)$, where $\kappa$ is 0.7 for females and 0.9 for males, $\alpha$ is -0.329 for females and -0.411 for males, min indicates the minimum of $\mathrm{SCr} / \kappa$ or 1 and max indicates the maximum of $\mathrm{SCr} / \kappa$ or 1 [18]. We defined hypertension as $\mathrm{BP} \geq 140 / 90 \mathrm{mmHg}$ or as being on anti-hypertensive medications.

\section{Statistical Analysis}

All data were analyzed using the IBM SPSS Statistics 24.0 program (IBM Corp., Armonk, NY). Values were reported as the mean \pm standard deviation, median with interquartile range or percentage, as appropriate. Data for all continuous variables were tested for normality. Skewed variables and all delta changes were logarithmically transformed before analysis. Data at baseline and at 26 weeks after initiation of SGLT2i therapy were compared using paired Student's $t$ test and the Wilcoxon signed-rank test. Pearson's correlations analysis was performed to determine the correlation of the reduction in serum ALT levels with baseline and changes of other variables after treatment with SGLT2i for 6 months. Multiple linear regression was performed to evaluate the independent variables that were associated with the reduction in serum ALT levels. One-way analysis of variance and the Chi-square test, or Fisher's exact test, whichever was appropriate, were also employed to compare if there was any difference between the use of dapagliflozin and empagliflozin, as well as between groups with or without background insulin therapy. In all statistical tests, two-sided $p$ values of $<0.05$ were considered to be significant.

\section{RESULTS}

\section{SGLT2i Improved Metabolic Parameters Among Chinese Participants with T2DM}

Of the 115 Chinese participants with T2DM included in this study, 69 and 46 were treated with dapagliflozin and empagliflozin, respectively. The mean age of the participants was $56.3 \pm 11.1$ years and mean duration of diabetes was $16.1 \pm 8.0$ years. Among them, $79.1 \%$ were non-drinkers or social drinkers, and $14.8 \%$ were ex-drinkers. With regard to background antidiabetic agents before starting on SGLT2i, 110 (95.7\%) participants were on metformin, 44 $(38.3 \%)$ were on dipeptidyl-peptidase- 4 inhibitors, $44(38.3 \%)$ were on sulphonylureas, six $(5.2 \%)$ were on glitazones, seven $(6.1 \%)$ were on glucagon-like-peptide- 1 receptor agonists and $66(57.4 \%)$ were on insulin therapy (Electronic Supplementary Material Table 1). At baseline, their mean BMI was $30.5 \pm 5.8 \mathrm{~kg} / \mathrm{m}^{2}$, mean HbA1c was $8.6 \pm 1.4 \%$ and fasting glucose was $9.1 \pm 2.7 \mathrm{mmol} / \mathrm{L}$. In addition, $102(88.7 \%)$ participants had hypertension, 76 (66.1\%) were on lipid-lowering medications and 32 (27.8\%) had established cardiovascular disease.

After 6 months of treatment with SGLT2i, metabolic parameters, including BW, SBP, fasting glucose and HbA1c, had improved significantly (Table 1 ). The participants showed a weight loss of $1.6 \mathrm{~kg}$ [95\% confidence interval (CI) -2.2 to $-1.1 ; p<0.001]$, reduction in SBP of $5.3 \mathrm{mmHg}(95 \% \mathrm{CI}-9.1$ to $-1.5 ; p=0.006)$, fasting glucose of $1.9 \mathrm{mmol} / \mathrm{L}(95 \% \mathrm{CI}-2.5$ to - 1.4; $p<0.001)$ and $\mathrm{HbA} 1 \mathrm{c}$ of $1.0 \%$ (95\% CI -1.2 to $-0.8 ; p<0.001$ ), as well as significantly increased high-density lipoprotein and decreased eGFR, at 26 weeks (HDL: mean change $0.04 \mathrm{mmol} / \mathrm{L}, \quad 95 \%$ CI $0.003-0.08$, $p=0.033$; eGFR: mean change $-1.6 \mathrm{ml} / \mathrm{min} /$ $1.73 \mathrm{~m}^{2}, 95 \%$ CI -2.9 to $-0.3, p=0.016$ ). Notably, among the 66 participants on background insulin therapy, after treatment with 
Table 1 Changes in metabolic parameters and serum alanine aminotransferase levels before and after 6 months of treatment with dapagliflozin and empagliflozin

\begin{tabular}{|c|c|c|c|c|}
\hline \multirow[t]{2}{*}{ Patient parameters } & \multicolumn{2}{|c|}{ Measurement time point } & \multirow{2}{*}{$\begin{array}{l}\text { Mean change from } \\
\text { baseline }(95 \% \mathrm{CI})\end{array}$} & \multirow[t]{2}{*}{$p$ value } \\
\hline & Baseline & $\begin{array}{l}6 \text { months post } \\
\text { treatment initiation }\end{array}$ & & \\
\hline $\mathrm{BW}(\mathrm{kg})$ & $81.1 \pm 18.1$ & $79.5 \pm 18.1$ & $-1.6(-2.2,-1.1)$ & $<0.001^{*}$ \\
\hline $\operatorname{BMI}\left(\mathrm{Kg} / \mathrm{m}^{2}\right)$ & $30.5 \pm 5.76$ & $29.9 \pm 5.75$ & $-0.6(-0.8,-0.4)$ & $<0.001^{*}$ \\
\hline SBP (mmHg) & $138 \pm 19.0$ & $132 \pm 18.0$ & $-5.3(-9.1,-1.5)$ & $0.006^{*}$ \\
\hline $\mathrm{DBP}(\mathrm{mmHg})$ & $77.4 \pm 10.5$ & $75.9 \pm 11.2$ & $-1.5(-3.7,0.6)$ & 0.155 \\
\hline $\mathrm{FPG}(\mathrm{mmol} / \mathrm{L})$ & $9.12 \pm 2.73$ & $7.20 \pm 1.91$ & $-1.9(-2.5,-1.4)$ & $<0.001^{*}$ \\
\hline HbA1c (\%) & $8.56 \pm 1.35$ & $7.55 \pm 0.90$ & $-1.0(-1.2,-0.8)$ & $<0.001^{*}$ \\
\hline $\operatorname{ALT}(\mathrm{U} / \mathrm{L})$ & $40.3 \pm 28.0$ & $29.0 \pm 14.1$ & $-11.3(-15.1,-7.6)$ & $<0.001^{*}$ \\
\hline $\operatorname{AST}(\mathrm{U} / \mathrm{L})$ & $28.2 \pm 13.2$ & $23.1 \pm 7.44$ & $-5.1(-7.1,-3.1)$ & $<0.001^{*}$ \\
\hline Albumin $(\mathrm{g} / \mathrm{L})$ & $43.3 \pm 3.11$ & $43.9 \pm 3.05$ & $0.6(0.1,1.1)$ & $0.017^{*}$ \\
\hline $\mathrm{TC}(\mathrm{mmol} / \mathrm{L})$ & $3.95 \pm 0.87$ & $3.96 \pm 0.85$ & $0.01(-0.12,0.13)$ & 0.887 \\
\hline $\mathrm{TG}(\mathrm{mmol} / \mathrm{L})$ & $1.84 \pm 1.43$ & $1.68 \pm 1.20$ & $-0.16(-0.34 .0 .03)$ & 0.098 \\
\hline $\mathrm{HDL}(\mathrm{mmol} / \mathrm{L})$ & $1.10 \pm 0.29$ & $1.14 \pm 0.27$ & $0.04(0.003,0.08)$ & $0.033^{*}$ \\
\hline $\mathrm{LDL}(\mathrm{mmol} / \mathrm{L})$ & $2.10 \pm 0.69$ & $2.07 \pm 0.67$ & $-0.03(-0.13,0.07)$ & 0.572 \\
\hline $\mathrm{eGFR}\left(\mathrm{ml} / \mathrm{min} / 1.73 \mathrm{~m}^{2}\right)$ & $91.3 \pm 19.6$ & $89.7 \pm 20.6$ & $-1.6(-2.9,-0.3)$ & $0.016^{*}$ \\
\hline
\end{tabular}

95\% CI 95\% confidence interval, $B W$ body weight, $B M I$ body mass index, SBP systolic blood pressure, DBP diastolic blood pressure, $F G$ fasting glucose, $H b A 1 c$ glycated hemoglobin, $A L T$ serum alanine aminotransferase, $A S T$ serum aspartate aminotransferase, $T C$ total cholesterol, $T G$ triglyceride, $H D L$ high-density lipoprotein, $L D L$ low-density lipoprotein, $e G F R$ estimated glomerular filtration rate

*Significant difference $(p<0.05)$ between data at baseline and at 26 weeks after initiation of sodium-glucose co-transporter 2 inhibitor (SGLT2i) therapy

SGLT2i, they showed a significant improvement in BW, fasting glucose and HbA1c despite a significant reduction in total insulin daily dose of $2.3 \mathrm{U}(95 \% \mathrm{CI}-4.2$ to $-0.4 ; p=0.019)$.

\section{SGLT2i Ameliorated Hepatic Dysfunction Among Chinese Participants with T2DM}

Participants treated with SGLT2i for 6 months also had a significant reduction in their mean serum ALT levels from $40.3 \pm 28.0$ to $29.0 \pm 14.1 \mathrm{U} / \mathrm{L}(p<0.001)$ (Table 1$)$, and the results were consistent among various subgroups, whether with or without background insulin therapy, and whether dapagliflozintreated or empagliflozin-treated (Fig. 1) The serum AST levels also decreased significantly from $28.2 \pm 13.2$ to $23.1 \pm 7.5 \mathrm{U} / \mathrm{L}(p<0.001)$, and the serum albumin increased significantly from $43.3 \pm 3.1$ to $43.9 \pm 3.1 \mathrm{U} / \mathrm{L}(p=0.017)$ after treatment with SGLT2i. Pearson's correlation analysis revealed a positive correlation between the reduction in serum ALT levels after SGLT2i treatment and baseline BW $(r=0.231$; $p=0.014), \quad$ log-transformed ALT $(r=0.747$; $p<0.001)$ and eGFR levels $(r=0.230$; $p=0.014)$, suggesting that participants who had higher BW and serum ALT and eGFR levels at baseline experienced a significantly greater improvement in serum ALT levels after 6 months of treatment with SGLT2i. Moreover, the reduction in serum ALT levels after 6 

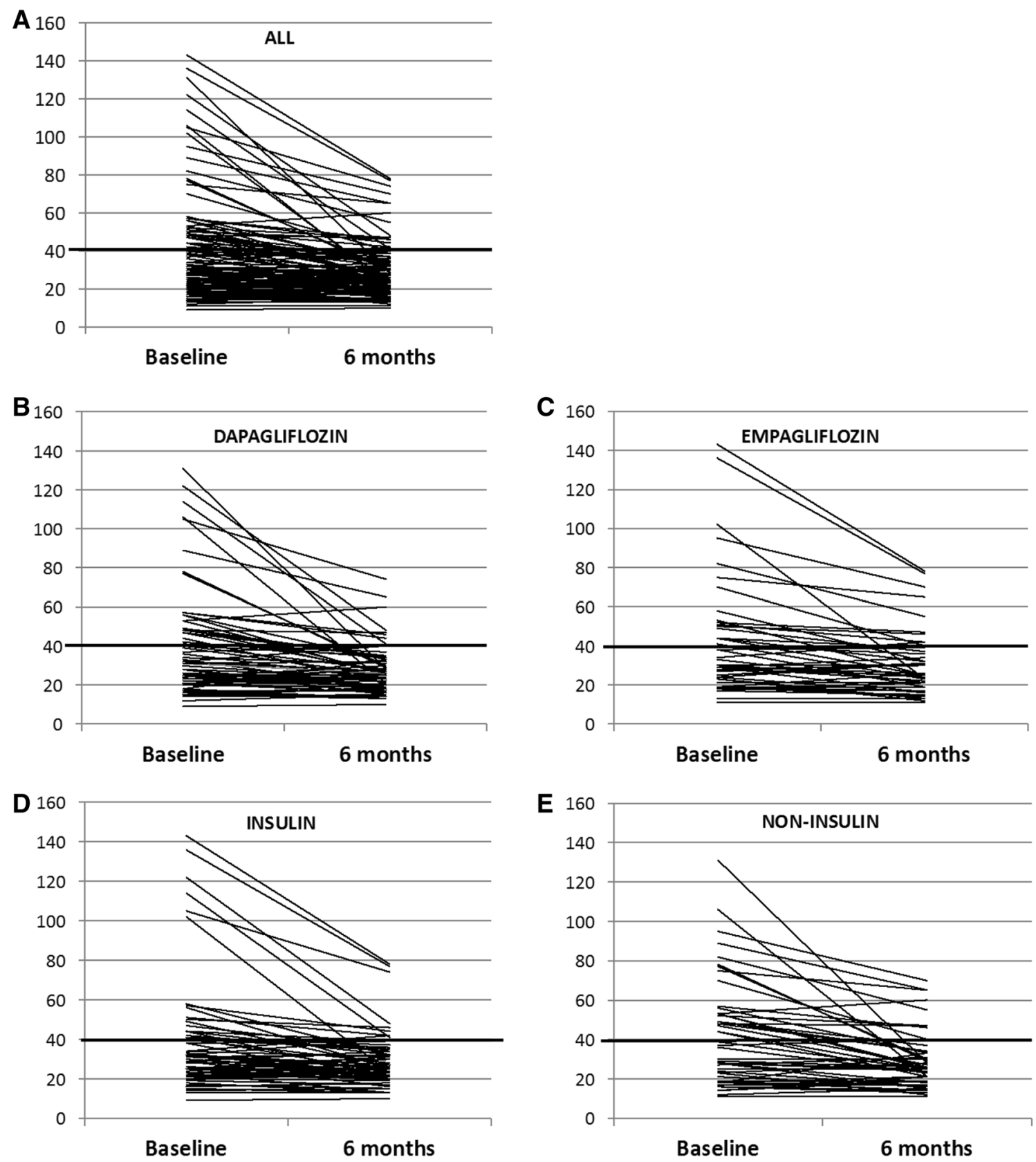

Fig. 1 Change in serum alanine aminotransferase levels from baseline to 6 months after initiation of treatment with dapagliflozin or empagliflozin. a All participants,

months of SGLT2i treatment also correlated positively with log-transformed changes in AST $(r=0.465 ; \quad p=<0.001), \quad$ fasting glucose $(r=0.248 ; p=0.014)$ and HbA1c $(r=0.192$; $p=0.043)$ but not with log-transformed changes in BW, SBP, serum albumin or eGFR levels

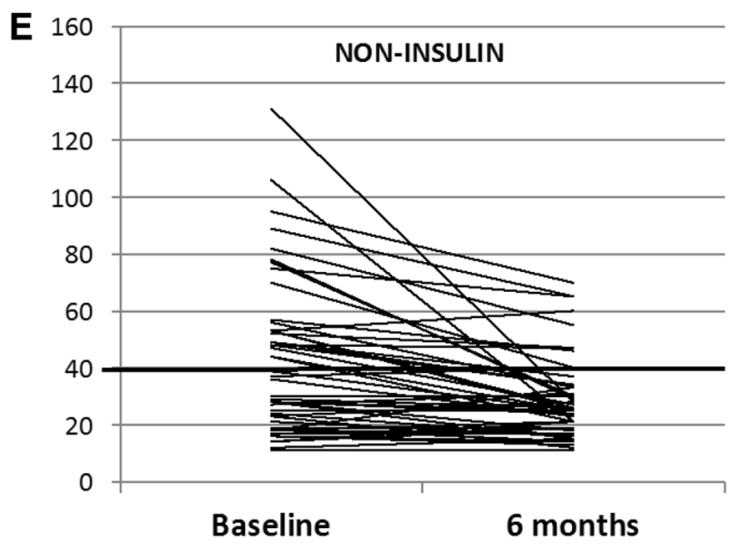

b participants on dapagliflozin, c participants on empagliflozin, d participants on insulin, e participants not on insulin)

(Table 2) The improvements in serum ALT levels after 6 months did not differ statistically between participants treated with dapagliflozin versus empagliflozin $(p=0.815)$, nor between participants with and without background insulin therapy $(p=0.429)$. Since the changes 
Table 2 Pearson's correlation analysis showing the correlation of the reduction in serum alanine aminotransferase levels with baseline and changes in other variables at 6 months of treatment

\begin{tabular}{|c|c|c|}
\hline Patients parameters & $R$ & $p$ value \\
\hline \multicolumn{3}{|l|}{ Baseline variables } \\
\hline BW & 0.231 & $0.014^{*}$ \\
\hline $\mathrm{ALT}^{\mathrm{a}}$ & 0.614 & $<0.001^{*}$ \\
\hline $\operatorname{AST}^{\mathrm{a}}$ & 0.401 & $<0.001^{*}$ \\
\hline Albumin ${ }^{\mathrm{a}}$ & 0.088 & 0.381 \\
\hline HbAlc & 0.007 & 0.938 \\
\hline FPG & -0.026 & 0.795 \\
\hline SBP & 0.095 & 0.325 \\
\hline $\operatorname{eGFR}\left(\mathrm{ml} / \mathrm{min} / 1.73 \mathrm{~m}^{2}\right)$ & 0.216 & $0.023^{*}$ \\
\hline \multicolumn{3}{|c|}{ Change from baseline variables ${ }^{\mathrm{a}}$} \\
\hline BW & 0.031 & 0.743 \\
\hline AST & 0.465 & $<0.001^{*}$ \\
\hline Albumin & 0.083 & 0.411 \\
\hline HbAlc & 0.192 & $0.043^{*}$ \\
\hline FPG & 0.248 & $0.014^{*}$ \\
\hline SBP & 0.003 & 0.978 \\
\hline $\mathrm{eGFR}\left(\mathrm{ml} / \mathrm{min} / 1.73 \mathrm{~m}^{2}\right)$ & -0.150 & 0.122 \\
\hline
\end{tabular}

* Significant difference at $p<0.05$

${ }^{a}$ Log-transformed before analysis

in AST and ALT levels were highly correlated $(r=0.465, p<0.001)$, as were the changes in the fasting glucose and HbA1c $(r=0.448$, $p<0.001)$, to avoid multi-collinearity, in the multiple linear regression analysis, either the change in fasting glucose or HbA1c was included in the model. Nonetheless, two separate analyses (Table 3) showed that the reduction in serum ALT levels was independently associated with the changes in both HbA1c and fasting glucose $(p=0.043$ for change in HbA1c and $p=0.014$ for change in fasting glucose), but not with the changes in other clinical variables, including BW. Using either the conventional serum ALT cutoff of $40 \mathrm{U} / \mathrm{L}[19,20]$ or the cutoff of $30 \mathrm{U} / \mathrm{L}$ as suggested by some authors for Asians $[20,21]$, there was a significant increase in participants with normal ALT levels after 6 months of treatment with SGLT2i, compared with baseline (Fig. 2).

\section{DISCUSSIONS}

To our knowledge, our study is the first to explore the relationship between the amelioration of hepatic dysfunction and the improvement in various metabolic parameters brought about by dapagliflozin and empagliflozin among Chinese subjects with T2DM. Although SGLT2i exert a glucose-lowering effect together with weight loss, both of which are beneficial in the management of NAFLD, our findings demonstrated that dapagliflozin and empagliflozin reduced serum ALT levels as a class effect, at least in part through an improvement in glycemia, but not through BW reduction, as reflected by the significant correlation between ALT reduction and changes in fasting glucose and HbA1c levels, but not with the change in BW.

SGLT2i have been shown to reduce serum ALT levels in clinical trials, although the patients in these trials were mostly from Western populations. A recent meta-analysis of 43 randomized controlled trials reported that serum ALT levels fell by $0.21 \mathrm{IU} / \mathrm{L}$ (95\% CI 0.33-0.10) after treatment with SGLT2i across all included studies [14]. However, the mechanism by which SGLT2i ameliorated hepatic dysfunction in T2DM remains controversial. Based on the results of their pooled analysis of data from four placebo-controlled and two active-controlled studies of canagliflozin, Leiter et al. attributed the significant improvement in serum ALT levels to combined HbA1c and body $\mathrm{BW}$ reduction, further noting that each $1 \%$ reduction in BW and $\mathrm{HbA1c}$ was associated with a 2 and $7 \%$ improvement in serum ALT levels, respectively [15]. Our findings are in line with their results with regard to the contribution of glycemic improvement towards the amelioration of hepatic dysfunction being much more important than BW reduction. A number of other animal and human studies using other 
Table 3 Multiple linear regression analysis showing the association between the reduction in serum alanine aminotransferase levels and change in glycated hemoglobin and fasting glucose levels, respectively, and other clinical variables after 6 months

\begin{tabular}{|c|c|c|c|c|c|c|}
\hline \multirow{2}{*}{$\begin{array}{l}\text { Changes in clinical } \\
\text { variables }^{\mathrm{a}}\end{array}$} & \multicolumn{3}{|l|}{ Model 1} & \multicolumn{3}{|l|}{ Model 2} \\
\hline & $\begin{array}{l}\text { Standardized } \\
\text { beta }\end{array}$ & Beta $(95 \% \mathrm{CI})$ & $p$ value & $\begin{array}{l}\text { Standardized } \\
\text { beta }\end{array}$ & Beta $(95 \% \mathrm{CI})$ & $p$ value \\
\hline \multicolumn{7}{|l|}{ HbAlc in Model } \\
\hline Weight & 0.007 & $0.067(-1.711,1.845)$ & 0.940 & - & - & - \\
\hline Albumin & 0.122 & $0.713(-0.400,1.826)$ & 0.207 & - & - & - \\
\hline HbAlc & 0.190 & $0.510(0.001,1.018)$ & $0.049^{*}$ & 0.192 & $0.514(0.017,1.011)$ & $0.043^{*}$ \\
\hline SBP & -0.008 & $-0.019(-0.481,0.443)$ & 0.936 & - & - & - \\
\hline eGFR & -0.149 & $-0.535(-1.219,0.148)$ & 0.124 & - & - & - \\
\hline \multicolumn{7}{|c|}{ Fasting glucose in Model } \\
\hline Weight & -0.003 & $-0.026(-1.793,1.740)$ & 0.976 & - & - & - \\
\hline Albumin & 0.112 & $0.656(-0.443,1.755)$ & 0.239 & - & - & - \\
\hline FG & 0.230 & $0.252(0.048,0.456)$ & $0.016^{*}$ & 0.232 & $0.255(0.053,0.456)$ & $0.014^{*}$ \\
\hline Systolic BP & 0.021 & $0.052(-0.405,0.508)$ & 0.822 & - & - & - \\
\hline eGFR & -0.155 & $-0.557(-1.233,0.119)$ & 0.105 & - & - & - \\
\hline
\end{tabular}

95\% CI 95\% confidence interval, HbA1c glycated hemoglobin, SBP systolic blood pressure, $e G F R$ estimated glomerular filtration rate, $F G$ fasting glucose

*Statistics test for comparision of ALT distribution between baseline and 6 months

${ }^{a}$ All variables were log-transformed before analysis

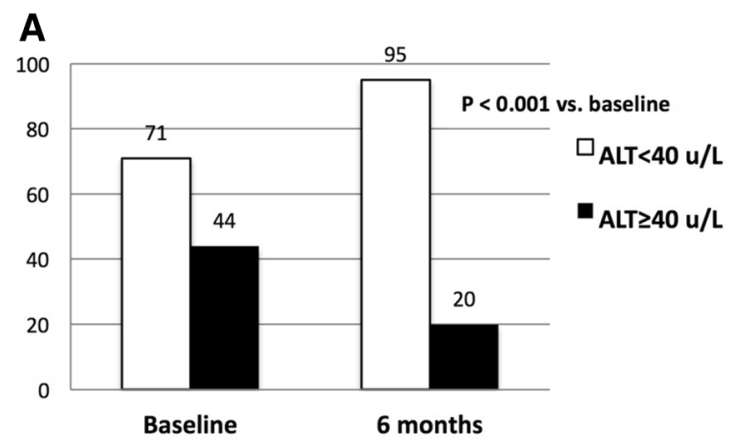

Fig. 2 Participants at baseline and after 6 months of treatment with dapagliflozin or empagliflozin, stratified by their serum alanine aminotransferase levels $(A L T)$. a ALT

SGLT2i also reported different findings from those observed in the pooled analysis of canagliflozin-although differences in sample size, study design and patient populations may

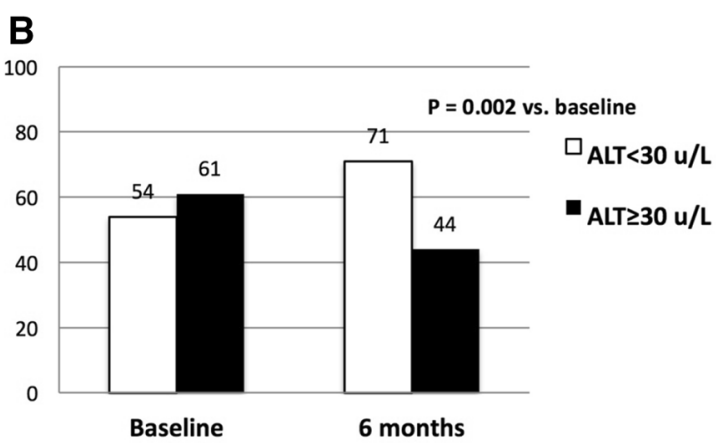

cutoff of $40 \mathrm{U} / \mathrm{L}, \mathbf{b}$ ALT cutoff of $30 \mathrm{U} / \mathrm{L}$; comparisons between baseline and 6 months ALT distributions by Fisher's exact test

explain (part of) the discrepancy in clinical outcomes. For example, in a mouse model of nonalcoholic steatohepatitis with diabetes, the use of empagliflozin decreased hepatic expression of 
several inflammatory genes, including tumor necrosis factor alpha, monocyte chemoattractant protein-1, interleukin-6 and suppressor of cytokine signaling- 3 , also independent of BW reduction [16]. In a recent randomized, activecontrolled trial involving human participants which compared the effects of pioglitazone and ipragliflozin on NAFLD, among the 32 subjects on ipragliflozin, despite their significant improvement in glycemic control, BW and subcutaneous and visceral fat after 24 weeks, the reductions in serum ALT levels correlated only with changes in BW but not with changes in visceral fat or HbA1c level [22]. On the other hand, in that same study, improvement in the liver-to-spleen attenuation ratio, which is a reflection of hepatic steatosis, correlated with changes in BW and $\mathrm{HbA} 1 \mathrm{c}$ and adiponectin levels [22]. However, in another Japanese study involving 48 Japanese subjects with T2DM, ipragliflozin was shown to reduce serum ALT levels irrespective of the reduction in BW, although the authors did not evaluate the relationship between glycemic improvement and the amelioration of hepatic dysfunction [17]. Taken together with the findings of the current study, these published data would suggest that the benefit of SGLT2i on hepatic dysfunction is likely to be a class effect, but the predominant effect through which SGLT2i ameliorate hepatic dysfunction in subjects with T2DM is still open to discussion. Theoretically, patients with NAFLD are required to lose up to $10 \%$ of their BW in order to improve necroinflammation, while a weight loss of 3-5\% might only improve hepatic steatosis [23]. In our study, subjects experienced a mean weight reduction of $1.6 \mathrm{~kg}$ over 6 months, in keeping with the generally expected weight loss of $1.5-3.5 \mathrm{~kg}$ reported among SGLT2i users over a period of 612 months [8]; this was equivalent to around 2\% of their baseline BW. Given this modest degree of weight loss brought by SGLT2i in our subjects, it may still be reasonable to suggest that the amelioration of hepatic dysfunction in our study population, as reflected by the improvement in liver enzyme levels, could be due to some other factors beyond weight reduction.

Our findings also highlight the role of glucotoxicity in modulating the progression of hepatic dysfunction. Chronic hyperglycemia can induce oxidative stress and endoplasmic reticulum stress responses and decrease AMPactivated protein kinase activity, hence promoting insulin resistance, hepatic steatosis and hepatocellular injury [24]. Although we found that the improvement in hepatic dysfunction significantly correlated with changes in glycemic parameters, the contribution of a direct effect of SGLT2i remains a possibility. In a randomized active-controlled trial of canagliflozin versus sitagliptin, despite a similar $\mathrm{HbA1c}$ improvement in both arms, there was a reduction in mean ALT levels among subjects randomized to canagliflozin $100 \mathrm{mg}$ that was not observed among those randomized to sitagliptin [25]. Furthermore, dapagliflozin had recently been shown to increase circulating adiponectin and zinc-A2-glycoprotein levels in Chinese subjects with T2DM [26]. The authors in that study attributed their findings to peroxisome proliferator activated-receptor (PPAR) gamma activation. Nonetheless, the results of our study would support a role of hyperglycemia (and possibly secondary improvement in insulin resistance), in the amelrioation of hepatic dysfunction by SGLT2i, although this hypothesis warrants validation in further mechanistic studies.

Our study has several limitations. First, the sample size was relatively small. Second, results from histological or imaging studies were not available in the majority of our study subjects as confirmation of the cause of hepatic dysfunction. However, given the mild degree of hepatic dysfunction, it might be impractical to perform liver biopsy in all of our subjects. Furthermore, although ultrasound scan of the liver is a common tool to screen for NAFLD, this tool can only pick up hepatic steatosis of $\geq 30 \%$. Last but not least, due to the clinical audit nature of the study, the causal relationship between the improvement in hepatic dysfunction and the changes in clinical variables could not be ascertained. Moreover, fasting insulin levels were not measured and hence conventional measures of the insulin resistance like homeostasis model assessment of insulin resistance (HOMA-IR) could not be assessed. Nonetheless, compared to most existing studies that have 
examined the relationship between SGLT2i and hepatic dysfunction, the majority of which comprised subjects with a relatively shorter duration of diabetes and not on insulin therapy, our study provides real-world observational data to support the use of SGLT2i in subjects with hepatic dysfunction across different patient populations of T2DM.

\section{CONCLUSIONS}

The use of SGLT2i is a novel approach to glycemic control and is accompanied by multiple demonstrable cardio-metabolic and renal benefits beyond its glucose-lowering effect. Our study results provide further support to the recent suggestion of considering SGLT2 $i$ in subjects comorbid with T2DM and NAFLD. With SGLT2i becoming available to such an enormous diabetic population in China, we have provided timely evidence that among Chinese subjects with T2DM, with or without background insulin therapy, dapagliflozin and empagliflozin not only improved metabolic parameters, but they also ameliorated hepatic dysfunction as a class, in part through alleviation of hyperglycemia, independent of BW reduction. Further prospective studies with larger sample sizes are certainly warranted to provide mechanistic insights into the benefits of SGLT2i in reversing hepatic dysfunction of T2DM.

\section{ACKNOWLEDGEMENTS}

Funding. No funding or sponsorship was received for this study or publication of this article. The article processing charges were funded by the authors.

Author Contributions. Paul Chi Ho Lee and Yunjuan $\mathrm{Gu}$ researched the data and wrote the manuscript. Man Yi Yeung researched the data. Carol Ho Yi Fong performed statistical analyses. Yu Cho Woo, Wing Sun Chow, Kathryn Tan and Karen Siu Ling Lam critically reviewed and edited the manuscript. Karen Siu Ling Lam initiated and supervised the study and is the guarantor of this work and as such had full access to all the data in the study and takes responsibility for the integrity of the data and the accuracy of the data analysis.

Authorship. All named authors meet the International Committee of Medical Journal Editors (ICMJE) criteria for authorship for this manuscript, take responsibility for the integrity of the work as a whole, and have given final approval to the version to be published.

Disclosures. Paul Chi Ho Lee, Yunjuan Gu, Man Yi Yeung, Carol Ho Yi Fong, Yu Cho Woo, Wing Sun Chow, Kathryn Tan and Karen Siu Ling Lam have nothing to disclose.

Compliance with Ethics Guidelines. This study is a clinical audit and does not involve any new studies of human subjects performed by any of the authors.

Data Availability. The datasets during and/ or analyzed during the current study are available from the corresponding author on reasonable request.

Open Access. This article is distributed under the terms of the Creative Commons Attribution-NonCommercial 4.0 International License (http://creativecommons.org/licenses/ by-nc/4.0/), which permits any noncommercial use, distribution, and reproduction in any medium, provided you give appropriate credit to the original author(s) and the source, provide a link to the Creative Commons license, and indicate if changes were made.

\section{REFERENCES}

1. Younossi ZM, Koenig AB, Abdelatif D, Fazel Y, Henry L, Wymer M. Global epidemiology of nonalcoholic fatty liver disease-meta-analytic assessment of prevalence, incidence, and outcomes. Hepatology. 2016;64:73-84.

2. Wang L, Gao P, Zhang M, Huang Z, Zhang D, Deng $\mathrm{Q}$, et al. Prevalence and ethnic pattern of diabetes and prediabetes in China in 2013. JAMA. 2017;317(24):2515-23. 
3. Kwok R, Choi KC, Wong GL, Zhang Y, Chan HL, Luk AO, et al. Screening diabetic patients for nonalcoholic fatty liver disease with controlled attenuation parameter and liver stiffness measurements: a prospective cohort study. Gut. 2016;65(8):1359-68.

4. Adams LA, Harmsen S, St Sauver JL, Charatcharoenwitthaya P, Enders FB, Therneau T, et al. Nonalcoholic fatty liver disease increases risk of death among patients with diabetes: a community-based cohort study. Am J Gastroenterol. 2010;105(7):1567-73.

5. Younossi ZM, Gramlich T, Matteoni CA, Boparai N, McCullough AJ. Nonalcoholic fatty liver disease in patients with type 2 diabetes. Clin Gastroenterol Hepatol. 2004;2(3):262-5.

6. El-Serag HB, Hampel H, Javadi F. The association between diabetes and hepatocellular carcinoma: a systematic review of epidemiologic evidence. Clin Gastroenterol Hepatol. 2006;4(3):369-80.

7. Zoppini G, Fedeli U, Gennaro N, Saugo M, Targher G, Bonora E. Mortality from chronic liver diseases in diabetes. Am J Gastroenterol. 2014;109(7):1020-5.

8. Lam KS, Chow CC, Tan KC, Ma RC, Kong AP, Tong $\mathrm{PC}$, et al. Practical considerations for the use of sodium-glucose co-transporter type 2 inhibitors in treating hyperglycemia in type 2 diabetes. Curr Med Res Opin. 2016;32(6):1097-108.

9. Zinman B, Wanner C, Lachin JM, Fitchett D, Bluhmki E, Hantel S, et al. Empagliflozin, cardiovascular outcomes, and mortality in type 2 diabetes. N Engl J Med. 2015;373(22):2117-28.

10. Wanner C, Inzucchi SE, Zinman B. Empagliflozin and progression of kidney disease in type 2 diabetes. N Engl J Med. 2016;375(18):1801-2.

11. Neal B, Perkovic V, Mahaffey KW, de Zeeuw D, Fulcher G, Erondu N, et al. Canagliflozin and cardiovascular and renal events in type 2 diabetes. N Engl J Med. 2017;377:644-57.

12. Kosiborod M, Cavender MA, Fu AZ, Wilding JP, Khunti K, Holl RW, et al. Lower risk of heart failure and death in patients initiated on SGLT-2 inhibitors versus other glucose-lowering drugs: The CVDREAL Study. Circulation. 2017;136:249-59.

13. Bril F, Cusi K. Management of nonalcoholic fatty liver disease in patients with type 2 diabetes: a call to action. Diabetes Care. 2017;40(3):419-30.

14. Mazidi M, Rezaie P, Gao HK, Kengne AP. Effect of sodium-glucose cotransport- 2 inhibitors on blood pressure in people with type 2 diabetes mellitus: a systematic review and meta-analysis of 43 randomized control trials with 22528 patients. J Am Heart Assoc. 2017;6(6):pii: e004007. https://doi. org/10.1161/JAHA.116.004007.

15. Leiter LA, Forst T, Polidori D, Balis DA, Xie J, Sha S. Effect of canagliflozin on liver function tests in patients with type 2 diabetes. Diabetes Metab. 2016;42(1):25-32.

16. Jojima T, Tomotsune T, Iijima T, Akimoto K, Suzuki $\mathrm{K}$, Aso Y. Empagliflozin (an SGLT2 inhibitor), alone or in combination with linagliptin (a DPP-4 inhibitor), prevents steatohepatitis in a novel mouse model of non-alcoholic steatohepatitis and diabetes. Diabetol Metab Syndr. 2016;8:45.

17. Komiya C, Tsuchiya K, Shiba K, Miyachi Y, Furuke S, Shimazu N, et al. Ipragliflozin improves hepatic steatosis in obese mice and liver dysfunction in type 2 diabetic patients irrespective of body weight reduction. PLoS One. 2016;11(3):e0151511.

18. Levey AS, Stevens LA, Schmid CH, Zhang YL, Castro AF 3rd, Feldman HI, et al. A new equation to estimate glomerular filtration rate. Ann Intern Med. 2009; 150(9):604-12.

19. Kaplan MM. Alanine aminotransferase levels: what's normal? Ann Intern Med. 2002;137(1):49-51.

20. Choudhary NS, Saraf N, Saigal S, Gautam D, Lipi L, Soin AS. Estimation of normal values of serum transaminases based on liver histology in healthy Asian Indians. J Gastroenterol Hepatol. 2015;30(4):763-6.

21. Tanabe A, Tatsumi F, Okauchi S, Yabe H, Tsuda T, Okutani K, et al. Optimal cut-off value of alanine aminotransferase level to precisely estimate the presence of fatty liver in patients with poorly controlled type 2 diabetes. J Diabetes Investig. 2016;7(4):645-6.

22. Ito D, Shimizu S, Inoue $K$, Saito D, Yanagisawa $M$, Inukai $\mathrm{K}$, et al. Comparison of ipragliflozin and pioglitazone effects on nonalcoholic fatty liver disease in patients with type 2 diabetes: a randomized, 24-week, open-label, active-controlled trial. Diabetes Care. 2017;40:1364-72.

23. Chalasani N, Younossi Z, Lavine JE, Diehl AM, Brunt EM, Cusi $\mathrm{K}$, et al. The diagnosis and management of non-alcoholic fatty liver disease: practice guideline by the American Association for the Study of Liver Diseases, American College of Gastroenterology, and the American Gastroenterological Association. Am J Gastroenterol. 2012;107(6):811-26. 
24. Mota M, Banini BA, Cazanave SC, Sanyal AJ. Molecular mechanisms of lipotoxicity and glucotoxicity in nonalcoholic fatty liver disease. Metabolism. 2016;65(8):1049-61.

25. Lavalle-Gonzalez FJ, Januszewicz A, Davidson J, Tong C, Qiu R, Canovatchel W, et al. Efficacy and safety of canagliflozin compared with placebo and sitagliptin in patients with type 2 diabetes on background metformin monotherapy: a randomised trial. Diabetologia. 2013;56(12):2582-92.

26. Liao X, Wang X, Li H, Li L, Zhang G, Yang M, et al. Sodium-glucose cotransporter 2 (SGLT2) inhibitor increases circulating zinc-alpha2-glycoprotein levels in patients with type 2 diabetes. Sci Rep. 2016;6:32887. 